\title{
Rapid response team and hospital mortality in hospitalized patients
}

Boris Jung ${ }^{1,2 \dagger}$, Aurelien Daurat ${ }^{1 \dagger}$, Audrey De Jong ${ }^{1,2}$, Gerald Chanques ${ }^{1,2}$, Martin Mahul ${ }^{1}$, Marion Monnin ${ }^{1}$, Nicolas Molinari ${ }^{3}$ and Samir Jaber ${ }^{1,2^{*}}$

\begin{abstract}
Purpose: Although rapid response systems are known to reduce in-hospital cardiac arrest rate, their effect on mortality remains debated. The present study aimed to evaluate the effect of implementing an intensivist-led rapid response team (RRT) on mortality in hospitalized patients.

Methods: An implementation of an intervention and a comparison with retrospective data analysis were performed in the four hospitals of Montpellier regional healthcare centre, in France. An intensivist-led RRT was implemented on a 24/7 basis along with educational modules, publicity and bedside simulation-based training in only one of the four hospitals from January 2012 to June 2012. A single activation criterion (heart rate below 40/min or above 140/min, systolic blood pressure below $80 \mathrm{mmHg}$, cardiac arrest, respiratory rate below 8/min or above 30/min, pulse oximetry below $90 \%$ with $\mathrm{O}_{2}$ above $6 \mathrm{l} / \mathrm{min}$, respiratory distress in a tracheotomised patient, respiratory arrest, coma or sudden change in level of consciousness, seizure) allowed any caregiver to directly contact the RRT using a dedicated cell phone number. Patients over 18 years admitted for more than $24 \mathrm{~h}$ in the medical-surgical wards from July 2010 to December 2011 (pre-RRT period) and from July 2012 to December 2013 (RRT period) were included. The main outcome was unexpected mortality. Analyses of data from one RRT hospital and three control hospitals (no RRT hospital) were performed.
\end{abstract}

Results: RRT implementation was associated with a decrease in unexpected mortality rate in the hospital that implemented RRT (from 21.9 to 17.4 per 1000 discharges; $p=0.002$ ). Reduction in unexpected mortality associated with RRT implementation could be estimated at 1.5 lives saved per week in the RRT hospital. In the three other hospitals, mortality rate was not significantly modified (from 19.5 to 19.9 per 1000 discharges; $p=0.69$ ). Overall mortality decreased from 39.6 to 34.6 per 1000 discharges between the pre-RRT and RRT period in the RRT hospital $(p=0.012)$, but did not significantly change in the other hospitals. Patients in the RRT hospital were more frequently admitted to the intensive care unit (ICU) during the RRT period (45.8 vs 52.9 per 1000; $p=0.002$ ), and their sequential organ failure assessment (SOFA) score upon ICU admission significantly decreased from 7 (4-10) to 5 (2-9); $p<0.001$.

Conclusions: In the present retrospective study, implementation of an intensivist-led RRT along with educational modules, publicity and bedside simulation-based training was associated with a significant decrease in unexpected and overall mortality of inpatients.

*Correspondence: s-jaber@chu-montpellier.fr

${ }^{1}$ Intensive Care Unit, Department of Anesthesia and Critical Care Medicine, University of Montpellier, Saint Eloi Teaching Hospital, 80

Avenue Augustin Fliche, 34295 Montpellier, Cedex 5, France

Full author information is available at the end of the article

Take home message: The implementation of an intensivist-led rapid response team was associated with a decrease in both overall and unexpected mortality in a study performed in a French teaching hospital.

${ }^{\dagger}$ B. Jung and A. Daurat contributed equally to the study. 
Keywords: Medical emergency team, Rapid response team, Cardiac arrest, Patient safety

\section{Introduction}

In Europe, in-hospital cardiac arrest rate ranges from 1 to 5 per 1000 hospital discharges [1]. Traditionally, when a member of hospital staff witnesses a cardiac arrest, they call the cardiac arrest team to attend to this medical emergency. A rapid response system (RRS) is a hospitalbased system designed to allow any staff member to alert other staff for help when a patient's vital signs have fallen outside set criteria. An intensivist-led rapid response team (RRT) is one in which an intensive care unit (ICU) doctor is a member of the response to the alert. The purpose of RRSs, which have mainly been developed in Northern America, Australia and Scandinavia, is to identify high-risk hospital patients early so that serious adverse events can be prevented and outcome improved $[2,3]$. Two previous meta-analyses concluded that RRSs were associated with a reduction in cardiac arrest but that the effect on mortality was doubtful $[4,5]$. The lack of a definite impact on outcome may have been due to several methodological issues from insufficient utilization to delays to activate the RRS [6]. Despite this lack of evidence, RRSs have been widely adopted in Australia and North America during the last decade $[7,8]$. Implementation in Europe remains rare and this study is one of the first such initiatives published in mainland Europe [9]. Our regional healthcare centre comprises four different hospitals. Each hospital is equipped with an independent ICU and the distances between them require an ambulance if a patient needs to be transferred.

The aim of the study was to assess whether the implementation of an intensivist-led RRT available only in one hospital would be associated with a decrease in the incidence of cardiac arrest and an increase in overall and unexpected hospital mortality.

\section{Methods}

The Comité d'Organisation et de Gestion de l'Anesthésie Réanimation (COGAR), a university hospital ethics committee in Montpellier, France, reviewed and approved the study. The need for informed consent was waived on the basis that the intervention was a quality improvement initiative.

\section{Study design}

We studied the effect of an RRT implemented in one hospital and compared that to retrospective data from three control hospitals. We included all consecutive adult patients admitted to the regional healthcare centre for at least $24 \mathrm{~h}$ between July 2010 and December 2013.
We divided the cohort into three temporal cohorts: the pre-RRT period ( 18 months, from July 2010 to December 2011), the implementation period (6 months, from January 2012 to June 2012) and the RRT period (18 months from July 2012 to December 2013).

In order to examine in-hospital outcome among patients most likely to benefit from an RRT intervention, we excluded patients hospitalized in the long-term geriatric care facility, those receiving maternity care, patients in the rehabilitation day-care facility and mental health services [10]. In these excluded units, specific emergency responses exist and involve ambulances with emergency physicians and none of these patients were hospitalized in an area within reach of the RRT initiative team.

The reporting of this study follows the Strengthening the Reporting of Observational Studies in Epidemiology (STROBE) guidelines [11] and the REporting of studies Conducted using Observational Routinely-collected health Data (RECORD) Statement [12]. The data were collected using dedicated forms designed according to the in-hospital resuscitation Utstein style [13] in the post-implementation period in the RRT hospital and using the hospital database (Programme de Médicalisation des Systèmes d'Information, PMSI, Montpellier University Hospital) for the pre-implementation period in the RRT hospital. The hospital database was used for both pre- and post-implementation periods in the three non-RRT hospitals.

\section{Setting}

As routinely observed in French teaching hospitals, hospital wards had a nurse/patient ratio ranging from $1: 12$ in the daytime (6.30 a.m. -9 p.m.) to 1:20 at night (9 p.m.6.30 a.m.) and the same ratio of assistant nurses. All ICUs were organized according to a closed format system and covered by a full team of attendings, fellows and residents during daytime ( 8 a.m. -6 p.m.) and one attending and one resident in house during night-time. Respiratory therapists, physician assistants and nurse practitioners are professions that do not exist in France. During the study period, there was no change in admission criteria, nursing or medical care plan or equipment. The RRT hospital case mix was mostly represented by abdominal and transplant surgery, liver and gastroenterology clinics, internal medicine, dermatology and haematology wards while the non-RRT hospitals provided all other specialities including cardiology and cardiac surgery. The coronary care unit was considered as a general ward, as was a step-down unit that did not belong to an ICU 
department. The non-RRT hospitals were chosen by convenience because of the location of health centre.

\section{Implementation}

In the pre-RRT period, all ICU teams responded to codes and triage calls using a traditional pyramid set up, involving the bedside nurse paging the resident then the fellow in charge who escalated to the attending on call. Either the fellow or the attending would eventually call the ICU nurse station to request a triage or a code blue response. No record of activity was obtained in that period. A code blue was defined as any patient with an unexpected cardiac or respiratory arrest requiring resuscitation [14].

In the implementation period, the RRT was instigated as a plan-do-study-act project $[15,16]$ in only one of the hospitals as a pilot. A multidisciplinary group composed of three attending intensivists (BJ, GC, SJ), the St Eloi ICU head nurse and an ICU fellow developed an activation criteria list adapted from the existing literature (Table 1) $[17,18]$.

A single criterion on that list allowed any caregiver to directly contact the RRT using a dedicated phone number (Fig. 1). The RRT comprised an ICU resident and either an ICU fellow or an attending. An ICU nurse could be part of the team if requested by the attending. No extra funding or caregiver hiring was associated with this pilot initiative. The RRT was equipped with a crash cart that included a portable ventilator, a monitor, a capnometer and oxygen [19]. The RRT was expected to reach the scene within $5 \mathrm{~min}$ in case of a code blue response and within $20 \mathrm{~min}$ in other situations. The RRT was expected to communicate with the ward team using the SBAR (situation, background, assessment, recommendation) scheme for communication and could provide any procedure, drugs prescription, facilitate an ICU transfer or discuss an end of life issue [20]. During a 6-month period, the RRT activation criteria were presented to the medical and nursing teams that were in the St Eloi ICU perimeter. Presentation included displaying posters, bedside simulation-based training courses using manikins for ward residents, doctors and nurses, practical educational sessions and information through the local hospital newspaper.

\section{Data collection and outcome assessment}

The study is a retrospective evaluation of the RRT intervention in one hospital (RRT hospital) complemented with data from three other hospitals (non-RRT hospitals). RRT intervention data and ICU data (demographics and outcome) in the RRT hospital were retrospectively analysed. Non-RRT ICU data (demographics and outcome) and inpatient data in all hospitals were retrospectively collected from the hospital billing codes (Programme de Médicalisation des Systèmes d'Information, PMSI).
Patient illness acuity or case-mix index across the different hospitals and during the study was categorized on a scale ranging from 1 (mild) to 4 (severe) according to the recommendations of the French Technical Agency on Hospital Information [21]. This index takes into account the main diagnosis, length of hospital stay and complications that might occur during the hospital stay. A senior hospital database manager, blinded to the study design and the scientific rationale, provided the case-mix index and the inpatient mortality rates. A surgical case was defined as one in which any surgery took place during the hospital stay.

The main endpoint of the study was the unexpected mortality rate defined as hospital-wide non-DNR and non-palliative care unplanned death per 1000 discharges as previously reported [22, 23]. The main endpoint was chosen according to guidelines for medical emergency teams [14]. DNR/palliative care order could be made during the hospital stay by the attending on service or by an intensivist in close collaboration with the ward doctors. Secondary endpoints were overall mortality, cardiac arrest rate per 1000 discharges occurring outside the ICU, unplanned ICU admission rate per 1000 discharges from medical and surgical wards (available only in the RRT hospital ICU), do not resuscitate patient deaths, and hospital length of stay. A cardiac arrest was defined as a cardiac arrest that was resuscitated.

\section{Statistical analysis}

On the basis of unpublished personal data of the RRT hospital (about 15,000 admissions per year, with an unexpected mortality of 2.1 per 1000 discharges), we estimated the number of subjects required for this study using the Arcsin approximation method. Assuming a relative reduction of $20 \%$ in unexpected mortality with a $5 \% \alpha$ risk and a $\beta$ risk of $90 \%$, we estimated that 22,023 subjects per period were needed. Consequently, with a yearly basis of about 15,000 admissions in the medical-surgical wards of the St Eloi Hospital, we set the observation periods duration to 18 months each. Normally distributed quantitative data were described as mean \pm standard deviation (SD) and compared using Student's $t$ test. If not normally distributed, quantitative data were expressed as median and interquartile range (IQR) and compared using the Mann-Whitney test. Qualitative data were expressed as number (rate) and compared using the Chi square test with Yates' correction as appropriate. Endpoints (monthly unexpected and overall mortality, cardiac arrest rate) were compared pre-RRT implementation (18-month period) and post-RRT implementation (18-month period) in the RRT hospital using a Mann-Whitney test. To limit the risk of reporting outcome results related to natural evolution or chance, we also compared the same endpoints 


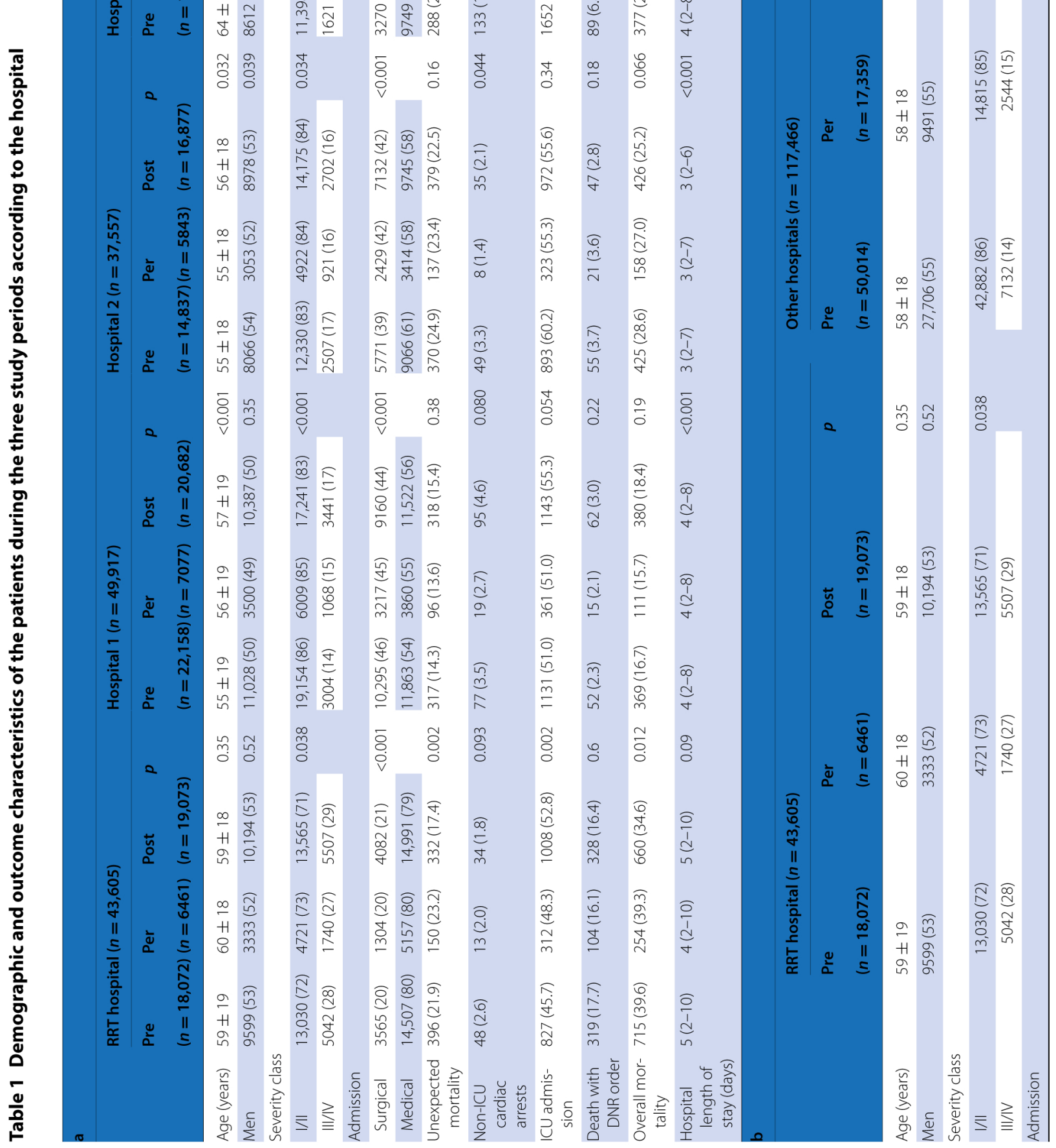


between the two 18-month periods in the three non-RRT hospitals [24]. Because the case mix was different among the four hospitals, an adjustment was made on the basis of the average age, gender and the average case-mix index using a linear and mixed regression model taking into account the month of hospitalization. A Poisson regression was also performed taking into account age, gender and severity index and the number of patients per month as offset variable to estimate the number of patients needed to be admitted in wards to save one unexpected death. Statistical analysis was performed using R software (version 3.0.2).

\section{Results}

\section{Demographics}

During the whole study period, a total of 161,071 patients were admitted for $24 \mathrm{~h}$ or more in the medical-surgical wards of the four healthcare centre hospitals. There were 68,086 patients during the pre-RRT period, and 69,165 in the RRT period. Demographic characteristics of patients during the two study periods according to hospital and period are summarized in Table 1.

\section{Unexpected mortality}

The unexpected mortality rate for 1000 discharges significantly decreased from 21.9 to 17.4 between the pre-RRT and post-RRT periods $(p=0.002)$.

After adjustment, the RR was calculated as equal to 0.77 (95\% CI 0.61-0.99) (Table 1). Assuming this relative reduction the number of patients hospitalized in wards needed to save one life was 225 (95\% CI 130-3788), $p=0.04$. With an average of about 18,000 admission/ year in the RRT hospital and on average one RRT activation per day, our initiative was associated with 1.5 lives saved a week. In the meantime no significant change was noted in mortality rates in the three hospitals that did not implement RRT (Fig. 2). Unexpected hospital mortality (including ICU mortality) following sepsis decreased in the RRT hospital [109/2571 (4.2\%) before RRT implementation vs $82 / 2638$ (3.1\%) after RRT implementation, $p=0.03$ but not in the three others hospitals with no RRT. In the RRT hospital, among the 142 septic ward patients for whom an RRT was called, 49 were admitted to the ICU and 11 of them (22\%) ultimately died. No data was available before RRT implementation. Data on the distinction between planned vs unplanned ICU admissions were collected only in the RRT hospital. The SOFA upon admission for unplanned admissions was 7 (4-10) before RRT implementation and 5 (2-9), $p<0.01$ after RRT implementation. The ICU mortality rate for these unplanned patients was $121 / 690$ (17.5 \%) before RRT implementation and 150/820 (18.3\%; $p=0.71)$ after RRT implementation. 


\section{CLINICAL WARNING SIGNS}

\section{CIRCULATION}

$\checkmark$ Cardiac Arrest

$\checkmark$ Heart rate below $40 / \mathrm{min}$ or above $140 / \mathrm{min}$

$\checkmark$ Systolic Blood Pressure below $80 \mathrm{mmHg}$

\section{RESPIRATORY}

$\checkmark$ Respiratory Arrest

$\checkmark$ Acute Respiratory Failure (respiratory rate below $8 / \mathrm{min}$ or above $30 / \mathrm{min}$ )

$\checkmark$ Pulse Oxymetry (SpO2) below $90 \%$ with $\mathrm{O} 2$ above $61 / \mathrm{min}$

$\checkmark$ Respiratory Distress in a tracheotomised patient

\section{CONSCIOUSNESS}

$\checkmark$ Coma or sudden change in level of consciousness $\checkmark$ Seizure

b
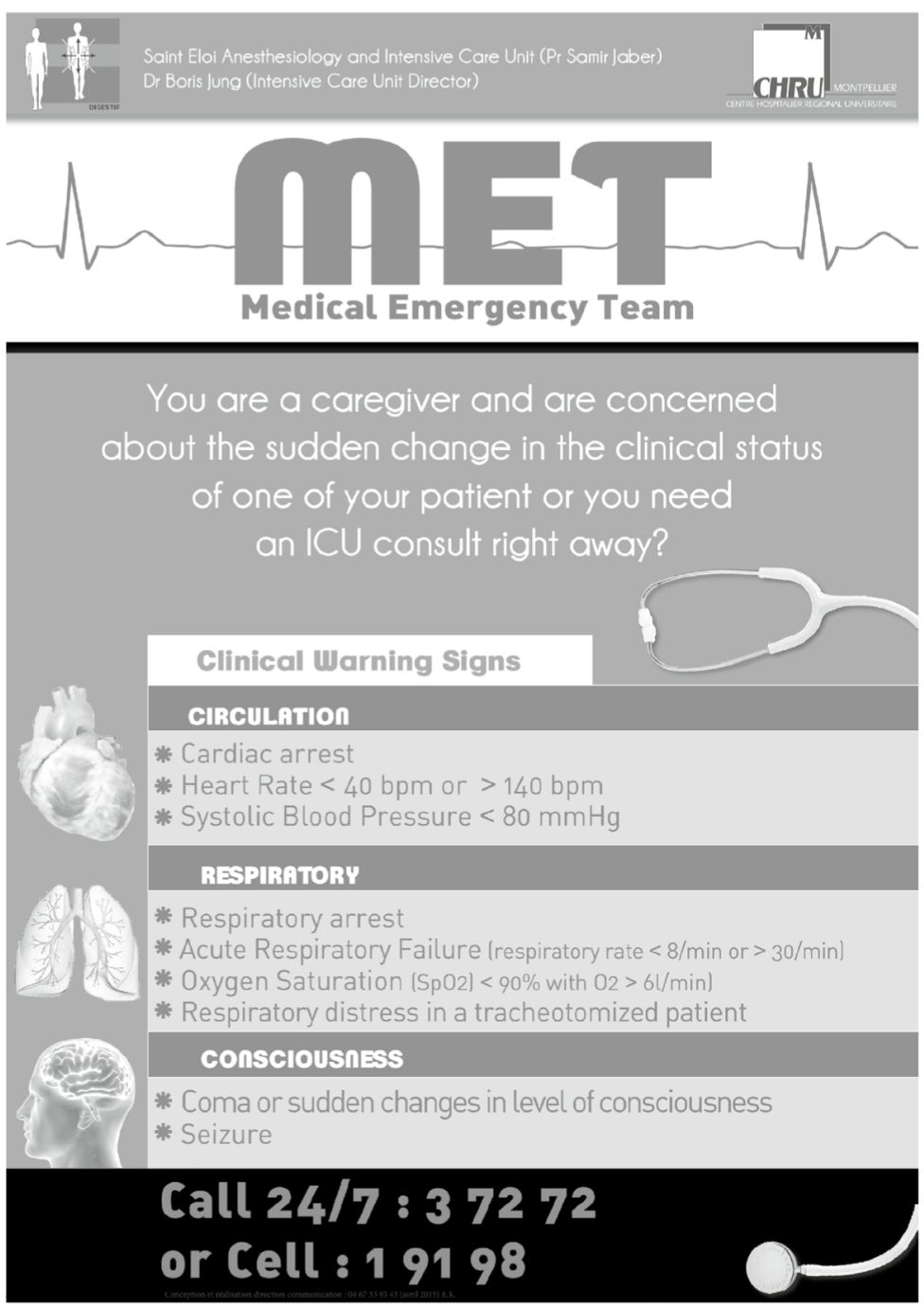

Fig. 1 a Activation criteria. A single criterion allowed any caregiver to directly reach the rapid response team (RRT) using a dedicated cell phone number. Because of their high-risk profile, the RRT could be activated following a subjective assessment in tracheotomised patients. b Poster reminding staff of the activation criteria and phone numbers of the medical emergency team 


\section{RRT hospital}
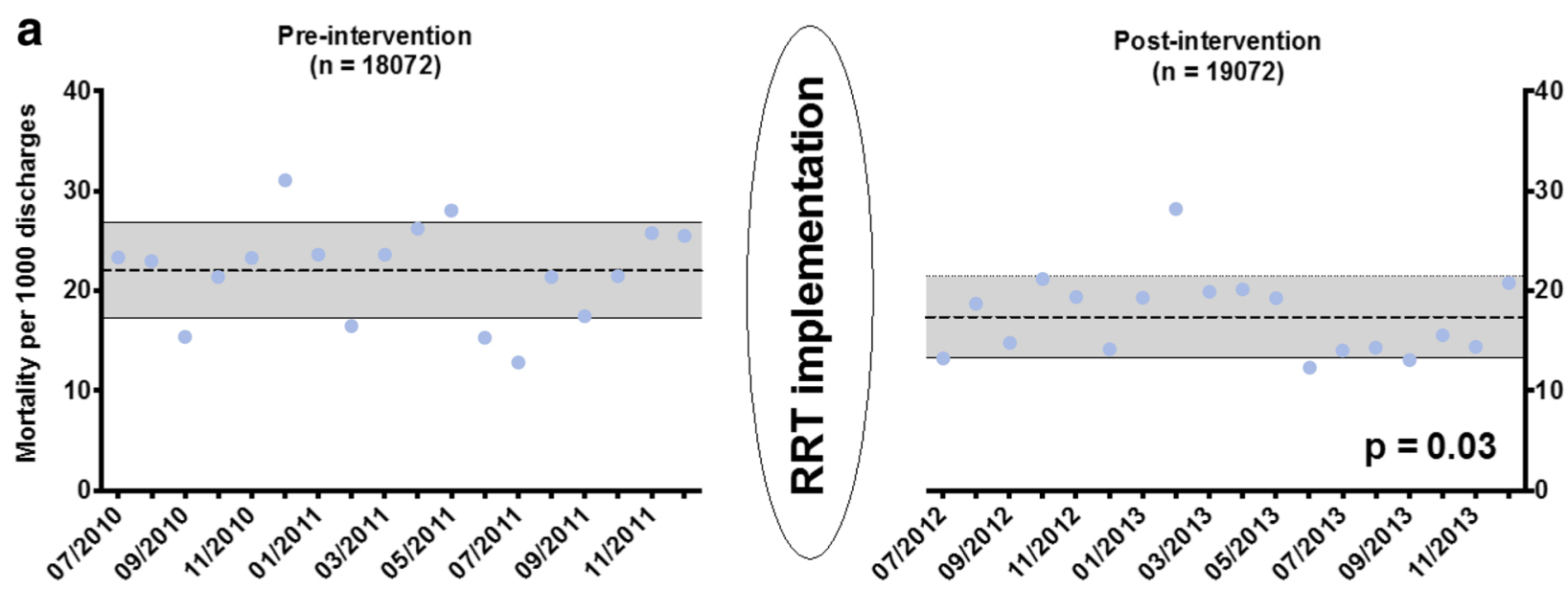

b

\section{Other hospitals}
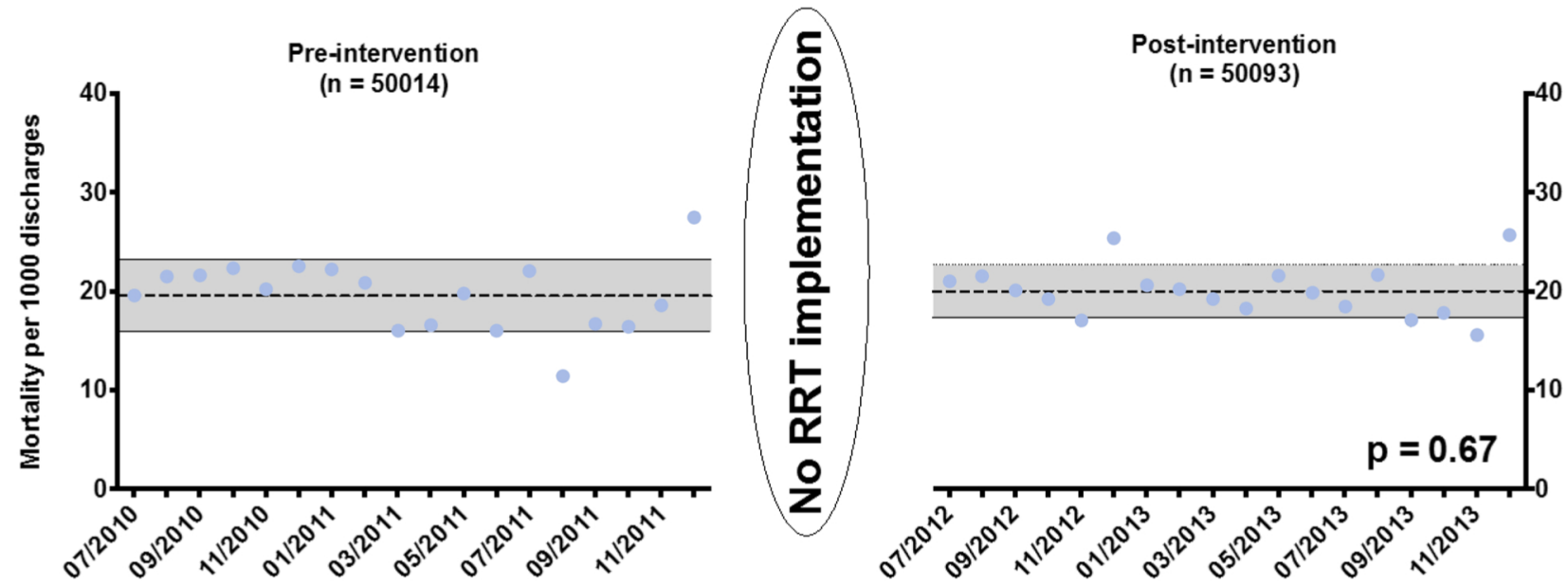

Fig. 2 Evolution of unexpected death rate per 1000 discharges by month in the RRT hospital (a), and in the three other hospitals (b). Dotted lines represent the mean rate per month. Grey rectangles represent the standard deviations

\section{Overall mortality and cardiac arrests}

Overall mortality decreased from 39.6 to 34.6 per 1000 discharges between the pre-RRT and RRT period in the RRT hospital $(P=0.012)$, but did not significantly change in the other hospitals (Table 1 ). We observed a decrease in cardiac arrest in the intervention period but this was not significant (2.6 vs 1.8 per 1000 admissions; $p=0.07$ ) so it could be due to chance alone. No trend in cardiac arrest incidence rate was observed in the three other hospitals (5.2 vs 5.3 per 1000 discharges, respectively in pre-RRT and RRT period, $p=0.84$ ). Rates of deaths with DNR orders and hospital length of stay did not change significantly between the two periods in any hospital (Table 1).

\section{RRT interventions}

During the 18-month RRT period, a total of 564 RRT interventions were carried out (i.e. 29.6 interventions per 1000 discharges). Median time of arrival was 5 min (5-10), and main activation criteria was acute hypoxaemia defined by $\mathrm{SpO}_{2}<90 \%$ (Table 2). The most common initial diagnosis was sepsis that accounted for $29 \%$ of the interventions (Table 2). Patients hospitalized in the RRT hospital during the RRT-period were more likely to be transferred to the ICU during their stay (pre-MET period 45.8 vs 52.9 per 1000 discharges in RRT period; $p=0.002)$, whereas rates of ICU admission did not statistically change in other hospitals (73.5 vs 72.7 per 1000 
discharges; $p=0.66$ ). In patients admitted to the ICU, median age significantly increased in the RRT hospital during the RRT period [59 (47-69) vs $61(50-71)$; $p=0.03$. In these patients, SOFA score was lower during the RRT period compared with the pre-RRT period: 7 (4-10) vs 5 (2-9); $p<0.001$. When planned admissions were not analysed, the SOFA score still decreased after the RRT implementation [7 (4-10) vs 5 (2-9); $p<0.01]$. In the RRT ICU, there was no overall significant change in SAPSII, ICU mortality, ICU length of stay and mechanical ventilation duration between the two study periods (Table 3 ).

\section{Discussion}

In this study, RRT implementation was associated with a $20.6 \%$ drop in unexpected mortality. In the meantime, the rate of ICU transfer from the wards increased and the organ failure score (SOFA) upon ICU admission of these patients decreased. None of these changes were noted in the three other hospitals that did not implement RRT. There was no significant difference between the two periods for cardiac arrest rate in any of the hospitals.

Until now, the effect of RRS on mortality has been debated with several discordant studies [25]. Two randomised clinical trials examined the impact of RRT on patient outcome $[26,27]$. In the large MERIT trial, 120,000 patients were enrolled in 23 hospitals in Australia but the mean calling rate in the RRT group was perhaps too low (8.7 calls per 1000 discharges) to have an impact on outcome. Moreover, as the RRT was part of a national initiative and was advertised in the media, the authors suspected a contamination effect of the control group. A second cluster-ward randomised trial performed in a British hospital evaluated a nurse-driven RRT and reported a positive effect on patient mortality [26], but the prospective cluster-ward design might have generated a Hawthorne effect. The meta-analysis by Chan et al. [28] reported a reduction in cardiac arrest rate but no significant effect on mortality [4]. More recently, the updated review by Winters et al. suggested a positive impact on patient outcome; however, statistical significance was not reached [5].

The RRT initiative in the present study meets quality criteria [14]: the "dose" of RRT delivered was 29.6 per 1000 patients, which is above the minimal rate of 25 per 1000 recommended [6], and median intervention time was short $(5 \mathrm{~min})$. The ward nurse staffing followed the French recommendations and the casemix index did not change between the study periods. We can, however, not speculate to what extent the RRT initiative would have been associated with an improved outcome if the nurse-patient ratio had been higher.
Table 2 Main activation criteria, primary actions, immediate triage decisions and bedside diagnosis after RRT intervention

\begin{tabular}{|c|c|}
\hline RRT activations & $564(100)$ \\
\hline \multicolumn{2}{|l|}{ RRT main activation criteria } \\
\hline $\mathrm{SpO}_{2}<90 \%$ & $97(17)$ \\
\hline $\mathrm{SBP}<80 \mathrm{mmHg}$ & $79(14)$ \\
\hline Altered mental status & $76(14)$ \\
\hline Respiratory rate $>30 \mathrm{c} / \mathrm{min}$ & $54(10)$ \\
\hline Dyspnoea & $45(8)$ \\
\hline Unspecific clinical concern & $36(6)$ \\
\hline Heart rate $>140$ bpm & $35(6)$ \\
\hline Cardiac arrest & $27(5)$ \\
\hline Haemorrhage & $21(4)$ \\
\hline Seizure & $13(2)$ \\
\hline Heart rate $<40$ bpm & $4(1)$ \\
\hline Others & $74(13)$ \\
\hline \multicolumn{2}{|l|}{ Position of activator calling RRT } \\
\hline Nurse & $167(30)$ \\
\hline Resident & $273(48)$ \\
\hline Fellow or attending & $105(19)$ \\
\hline Non medical staff & $19(3)$ \\
\hline \multicolumn{2}{|c|}{ Main primary action performed by RRT } \\
\hline Crystalloid infusion & $123(22)$ \\
\hline Antibiotics & $43(8)$ \\
\hline Peripheral line & $39(7)$ \\
\hline Intubation & $36(6)$ \\
\hline Colloid infusion & $36(6)$ \\
\hline Diuretics & $36(6)$ \\
\hline Strategy advice only & $32(6)$ \\
\hline Transfusion & $31(5)$ \\
\hline Compressions for acute bleeding & $25(4)$ \\
\hline Nebulizer treatment & $22(4)$ \\
\hline Vasopressor & $21(4)$ \\
\hline Antiarrhythmic treatment & $16(3)$ \\
\hline Non-invasive ventilation & $12(2)$ \\
\hline Defibrillation & $10(2)$ \\
\hline Flumazenil & $10(2)$ \\
\hline Central venous access & $6(1)$ \\
\hline Analgesics & $5(1)$ \\
\hline Antiepileptic drugs & $5(1)$ \\
\hline Others & $47(8)$ \\
\hline \multicolumn{2}{|l|}{ Immediate triage } \\
\hline Patient was not an ICU candidate & $348(62)$ \\
\hline Too sick to benefit & $66(12)$ \\
\hline Too well to benefit & $282(50)$ \\
\hline ICU admission & $187(33)$ \\
\hline Death after resuscitation & $19(4)$ \\
\hline Missing data & $10(2)$ \\
\hline \multicolumn{2}{|l|}{ Initial diagnosis } \\
\hline Sepsis & $142(25)$ \\
\hline Severe sepsis/sepsis shock & $121(21)$ \\
\hline
\end{tabular}




\section{Table 2 continued}

\begin{tabular}{lc}
\hline RRT activations & $564(100)$ \\
\hline Non severe sepsis & $11(2)$ \\
\hline Immunodepression-related sepsis & $10(2)$ \\
\hline Haemorrhage/haemorrhagic shock & $45(8)$ \\
\hline Hypovolaemia & $11(2)$ \\
\hline Acute heart failure/cardiogenic shock & $52(9)$ \\
Arrhythmia & $12(2)$ \\
\hline Pulmonary embolism & $11(2)$ \\
Acute on chronic liver failure & $38(7)$ \\
Acute pancreatitis & $11(2)$ \\
Stroke & $9(2)$ \\
\hline Seizure & $17(3)$ \\
Altered mentality & $4(1)$ \\
\hline Anaphylaxis & $15(3)$ \\
\hline COPD exacerbation & $15(3)$ \\
\hline Acute renal failure & $8(1)$ \\
\hline Orug overdose & $7(1)$ \\
\hline Missing data & $91(16)$ \\
\hline
\end{tabular}

Data are expressed as number and percentages of total RRT intervention $(n=564)$

COPD chronic obstructive pulmonary disease, $I C U$ intensive care unit, $R R T$ rapid response team, $\mathrm{SpO} 2$ oxyhaemoglobin saturation measured by pulse oximetry, $S B P$ systolic blood pressure

The team was led by an ICU physician which might have contributed to a greater efficiency [28]. Our initiative concerned one area of the regional healthcare centre while other areas depended on other ICUs with no RRT. To avoid daytime rounds and disruption of tasks, the $1-2$ activations per $24 \mathrm{~h}$ were mostly provided by attendings assigned to non-clinical duty and by the inhouse team on call during night-time. The RRT implementation was associated with a higher rate of early admission of less sick and older patients (Table 3), a double-edged sword effect linked to RRT systems [30, 31] but associated with a decrease in mortality at a minimal cost in the present study. Reduction of unexpected mortality associated with RRT implementation is partially related to a positive impact on outcome in patients diagnosed with sepsis. Our data are in line with the hypothesis that RRT might have taught caregivers to respond better to sepsis in patients admitted in wards but also provided a rapid ICU consultation to respond early to a patient presenting a progressing sepsis. This is also in line with extensive literature on early goal-directed therapy and the Surviving Sepsis Campaign that emphasize the need for rapid response in sepsis patients [32, 33].
Notably, RRT implementation was not associated with a statistically significant decrease in cardiac arrest rate in the present study possibly because of lack of power or because the rate was already low before the implementation. However, we observed a decrease in cardiac arrest rate in hospital 2 possibly because of a decrease in the patients' severity in this hospital (Table 1a).

The present study has several strengths. First, we enrolled a large number of patients (i.e. 137,251) over a consecutive 3-year-period and we performed the implementation of an RRT intervention (only RRT hospital) and a comparison with retrospective data analysis (both RRT and non-RRT hospitals). However, as the case mix was different among the four hospitals, we adjusted for patient's severity (based on the case-mix index), gender and age.

Several limitations can also be discussed. All planned and unplanned admissions were considered because this distinction was not available in the non-RRT hospitals. A single RRT activation parameter was used in this study, although aggregate weighted scoring systems may be more accurate but also less user-friendly [29]. We did not evaluate healthcare-related costs but the cost of the intervention may have been minimized by the fact that the RRT team was part of the ICU routine care without hiring extra staff. DNR orders and cardiac arrest rate were collected from the hospital electronic data reports in the four hospitals and could have been preceded by RRT activation. No modifications of ICU protocols were made that could have influenced the outcome of patients admitted after RRT activation but the ICU team in the RRT hospital was motivated to provide the best service including early ICU admission for high-risk patients. It would have been of interest to report the call rate during a fourth period to check if the call rate was sustained after implementation but data this was not collected. Motivation may be viewed as an interpretation bias but is necessary in any RRT implementation. It took significant efforts to launch the RRT initiative and to train ICU staff but also to convince ward doctors and nurses to contact the RRT for high-risk patients without delay. A training and educational module including bedside simulation with a manikin, information using posters and advertisement was designed to help caregivers, and the nurses in particular, to recognize the listed activation criteria.

\section{Conclusion}

Intensivist-led RRT implementation with educational modules for nurses and doctors, bedside simulationbased training and publicity was associated with a significant decrease in unexpected and overall mortality of hospitalized patients. 


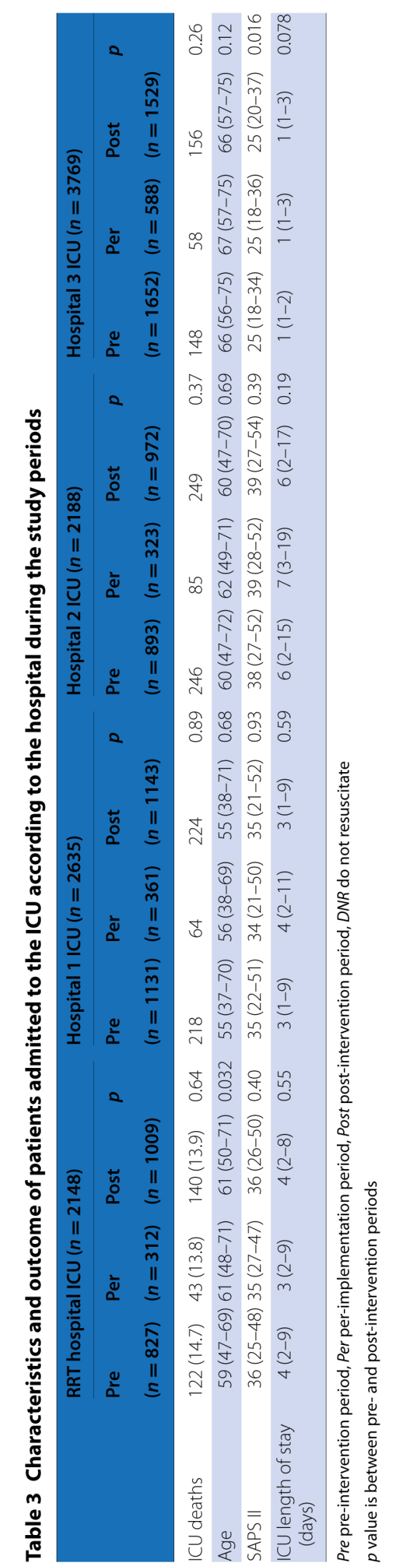




\section{Author details}

'Intensive Care Unit, Department of Anesthesia and Critical Care Medicine, University of Montpellier, Saint Eloi Teaching Hospital, 80 Avenue Augustin Fliche, 34295 Montpellier, Cedex 5, France. ${ }^{2}$ Centre National de la Recherche Scientifique (CNRS 9214), Institut National de la Santé et de la Recherche Medicale (INSERM U-1046), Montpellier University, Montpellier, France. ${ }^{3}$ Department of Statistics, University of Montpellier Lapeyronie Hospital, UMR 729 MISTEA, Montpellier, France.

\section{Acknowledgments}

We thank the nurses and physicians of the rapid response team of the ICU of Saint Eloi Hospital for their work in collecting data and their support in the RRT implementation. The authors also acknowledge Valerie Maccioce for the English editing, David Demoulin for his invaluable help and support in extracting data from the hospital database and the Montpellier University Hospital Communication Department for having designed the flyers and poster.

\section{Compliance with ethical standards}

\section{Conflicts of interest}

Boris Jung reports personal fees from Merck (Whitehouse station, $\mathrm{NJ}$ ) and Astellas (Tokyo, Japan) not related to the present study. Aurelien Daurat, Audrey De Jong, Martin Mahul, Marion Monnin, Gerald Chanques and Nicolas Molinari have nothing to disclose. Samir Jaber reports personal fees from Maquet, Draeger, Hamilton Medical and Fisher Paykel not related to the present study.

\section{Funding}

This study was supported by the University Hospital of Montpellier

\section{References}

1. Nolan JP, Soar J, Cariou A et al (2015) European Resuscitation Council and European Society of Intensive Care Medicine guidelines for post-resuscitation care 2015: section 5 of the European Resuscitation Council guidelines for resuscitation 2015. Resuscitation 95:202-222. doi:10.1016/j.resuscitation.2015.07.018

2. Brennan TA, Leape LL, Laird NM et al (1991) Incidence of adverse events and negligence in hospitalized patients. Results of the Harvard Medical Practice Study I. N Engl J Med 324:370-376. doi:10.1056/ NEJM199102073240604

3. Zegers M, de Bruijne MC, de Keizer B et al (2011) The incidence, root-causes, and outcomes of adverse events in surgical units: implication for potential prevention strategies. Patient Saf Surg 5:13. doi:10.1186/1754-9493-5-13

4. Jones D, Bellomo R, DeVita MA (2009) Effectiveness of the medical emer gency team: the importance of dose. Crit Care 13:313. doi:10.1186/cc7996

5. England K, Bion JF (2008) Introduction of medical emergency teams in Australia and New Zealand: a multicentre study. Crit Care 12:151. doi:10.1186/cc6902

6. Institute for Healthcare Improvement (2014) Overview. http://www. ihi.org/Engage/Initiatives/Completed/5MillionLivesCampaign/Pages/ default.aspx. Accessed 9 Oct 2014

7. Hilton AK, Jones D, Bellomo R (2013) Clinical review: the role of the intensivist and the rapid response team in nosocomial end-of-life care. Crit Care 17:224. doi:10.1186/cc11856

8. Salvatierra G, Bindler RC, Corbett C et al (2014) Rapid response team implementation and in-hospital mortality. Crit Care Med 42:2001-2006. doi:10.1097/CCM.0000000000000347

9. von Elm E, Altman DG, Egger M et al (2007) The strengthening the reporting of observational studies in epidemiology (STROBE) statement: guidelines for reporting observational studies. Ann Intern Med 147:573-577. doi:10.7326/0003-4819-147-8-200710160-00010

10. Benchimol El, Smeeth L, Guttmann A et al (2015) The reporting of studies conducted using observational routinely-collected health data (RECORD) statement. PLoS Med 12:e1001885. doi:10.1371/journal.pmed.1001885

11. Cummins RO, Chamberlain D, Hazinski MF et al (1997) Recommended guidelines for reviewing, reporting, and conducting research on in-hospital resuscitation: the in-hospital "Utstein style". Circulation 95:2213-2239
12. Devita MA, Bellomo R, Hillman K et al (2006) Findings of the first consensus conference on medical emergency teams. Crit Care Med 34:24632478. doi:10.1097/01.CCM.0000235743.38172.6E

13. de Jong A, Molinari N, de Lattre S et al (2013) Decreasing severe pain and serious adverse events while moving intensive care unit patients: a prospective interventional study (the NURSE-DO project). Crit Care 17:R74. doi:10.1186/cc12683

14. Taylor MJ, McNicholas C, Nicolay C et al (2014) Systematic review of the application of the plan-do-study-act method to improve quality in healthcare. BMJ Qual Saf 23:290-298. doi:10.1136/bmjqs-2013-001862

15. Chan PS, Khalid A, Longmore LS et al (2008) Hospital-wide code rates and mortality before and after implementation of a rapid response team. JAMA 300:2506-2513. doi:10.1001/jama.2008.715

16. Subbe CP, Kruger M, Rutherford P, Gemmel L (2001) Validation of a modified early warning score in medical admissions. QJM 94:521-526

17. Jaber $S$, Jung $B$, Corne $P$ et al (2010) An intervention to decrease complications related to endotracheal intubation in the intensive care unit a prospective, multiple-center study. Intensive Care Med 36:248-255. doi:10.1007/500134-009-1717-8

18. Haig KM, Sutton S, Whittington J (2006) SBAR: a shared mental model for improving communication between clinicians. Jt Comm J Qual Patient Saf 32:167-175

19. ATIH (2015) MCO: Classification médico-économique. http://www.atih. sante.fr/mco/classification-medico-economique. Accessed 13 Apr 2015

20. Le Gall JR, Lemeshow S, Saulnier F (1993) A new simplified acute physiology score (SAPS II) based on a European/North American multicenter study. JAMA 270:2957-2963

21. Vincent $J L$, Moreno R, Takala J et al (1996) The SOFA (sepsis-related organ failure assessment) score to describe organ dysfunction/failure. On behalf of the working group on sepsis-related problems of the European Society of Intensive Care Medicine. Intensive Care Med 22:707-710

22. Hillman K, Chen J, Cretikos M et al (2005) Introduction of the medical emergency team (MET) system: a cluster-randomised controlled trial. Lancet 365:2091-2097. doi:10.1016/S0140-6736(05)66733-5

23. Al-Qahtani S, Al-Dorzi HM, Tamim HM et al (2013) Impact of an intensivist-led multidisciplinary extended rapid response team on hospitalwide cardiopulmonary arrests and mortality. Crit Care Med 41:506-517. doi:10.1097/CCM.0b013e318271440b

24. Zhu B, Li Z, Jiang L et al (2015) Effect of a quality improvement program on weaning from mechanical ventilation: a cluster randomized trial. Intensive Care Med 41:1781-1790. doi:10.1007/s00134-015-3958-z

25. Sandroni C, Caricato A (2013) Are rapid response systems effective in reducing hospital mortality? Crit Care Med 41:679-680. doi:10.1097/ cCM.0b013e318275cb7d

26. Priestley G, Watson W, Rashidian A et al (2004) Introducing critical care outreach: a ward-randomised trial of phased introduction in a general hospital. Intensive Care Med 30:1398-1404. doi:10.1007/s00134-004-2268-7

27. Hillman K, Chen J, Cretikos M et al (2005) Introduction of the medical emergency team (MET) system: a cluster-randomised controlled trial. Lancet 365:2091-2097. doi:10.1016/S0140-6736(05)66733-5

28. Chan PS, Jain R, Nallmothu BK et al (2010) Rapid response teams: a systematic review and meta-analysis. Arch Intern Med 170:18-26. doi:10.1001/archinternmed.2009.424

29. McNeill G, Bryden D (2013) Do either early warning systems or emergency response teams improve hospital patient survival? A systematic review. Resuscitation 84:1652-1667. doi:10.1016/j. resuscitation.2013.08.006

30. Winters BD, Weaver SJ, Pfoh ER et al (2013) Rapid-response systems as a patient safety strategy: a systematic review. Ann Intern Med 158:417-425. doi:10.7326/0003-4819-158-5-201303051-00009

31. Jones DA, DeVita MA, Bellomo R (2011) Rapid-response teams. N Engl J Med 365:139-146

32. Kumar A, Roberts D, Wood KE et al (2006) Duration of hypotension before initiation of effective antimicrobial therapy is the critical determinant of survival in human septic shock. Crit Care Med 34:1589-1596. doi:10.1097/01.CCM.0000217961.75225.E9

33. Dellinger RP, Levy MM, Rhodes A et al (2013) Surviving Sepsis Campaign: international guidelines for management of severe sepsis and septic shock: 2012. Crit Care Med 41:580-637. doi:10.1097/ CCM.0b013e31827e83af 